\title{
Analysis on Scheduling and Load Balancing Techniques in Wireless Mesh Networks
}

\author{
Jasmine Joseph \\ Post Graduate Scholar \\ Department of Information Technology \\ Karunya University, India
}

\author{
M. Princy \\ Lecturer \\ Department of Information Technology \\ Karunya University, India
}

\begin{abstract}
The Wireless Mesh Network (WMN) has become the focus of much research since they allow increased network coverage, low cost and easy deployment. WMN includes the Internet Gateways (IGW), mesh routers and mesh clients forming a three layer architecture. Throughput and fairness are major issues in WMN as the network needs to satisfy increasing network demands and must be fair to all the clients requesting the demand. The gateway may become a bottleneck as all the traffic in WMN is routed to and from the gateway. All these above factors affect the performance of network. Thus the scheduling and load-balancing have become main challenges in WMN. Several scheduling and load balancing schemes have been discussed in this paper. Two scheduling models explored are interference-based and tree-based. Four load balancing schemes discussed are path-based, gateway-based and mesh-router-based.
\end{abstract}

\section{General Terms}

Wireless mesh networks (WMN), throughput, fairness.

\section{Keywords}

WMN, scheduling, load balancing.

\section{INTRODUCTION}

Wireless mesh networks are replacing traditional wired networks. It provides broadband technology which ensures high bandwidth internet connection to a large geographical area with minimum deployment and maintenance cost. WMN is a collection of self-configurable, self healing mesh routers and clients. Mesh routers serve as a backbone to provide network access for mesh clients as the mesh routers form a multi-hop wireless network. In WMN, the network connection is spread out among hundreds of mesh clients that communicate with each other to share the network connection across large area. The applications using WMN includes broadband internet access, distributed information sharing and storage, various real-time multimedia applications. It seems that WMN is truly wireless, which is the most advantageous feature of it. Mesh routers have minimal stability with fixed AC power supply. This feature makes it different from wireless ad-hoc and wireless sensor networks.

Mesh routers can have both gateway and bridge functionality. It even contains additional routing functions to support mesh networking. Since it possesses multi-hop communication, it can achieve high coverage area with much lower transmission power. Because of gateway/bridge functionality of mesh router, various other networks can be integrated into the WMN. Mesh clients can also work as router but it does not have gateway/bridge functionalities. Because of the multi-hop communication as mentioned earlier, it is possible for hundreds of users to share a single broadband connection.

Some technical challenges in WMN are scheduling, optimal routing, load balancing, and network auto-configuration and mobility management. This work is focusing on scheduling and load balancing.

The rest of the paper is organized as follows: the important issues in WMN are discussed in section II. Section III describes the scheduling mechanisms, interference and treebased models. Section IV discusses on three different load balancing schemes. Analysis of all the scheduling and load balancing schemes are given in section $\mathrm{V}$.

\section{IMPORTANT ISSUES}

Throughput and fairness are the most critical issues in WMN. High throughput is necessary in order to meet the increasing demand of network applications. Fairness must be guaranteed among different clients who pay the same flat rate for services from the network. Thus scheduling is the main challenge faced by this network. Only the performance parameters like throughput, delay or some other features are being optimized in current deployments whereas fairness is given less concern [3]. Fair scheduling assigns transmission rights to the links so that the links can be activated simultaneously, thus improving spatial reuse [1,6] which will eventually leads to high throughput. Each user, requesting for service should get similar QoS.

Load balancing $[3,5,20]$ is one of the factors that affects both throughput and fairness of the network. As the purpose of WMN is to serve a huge community of users, the average volume of traffic is significantly higher than in typical MANET environment. Limiting the number of gateways to one can cause an extreme bottleneck at this gateway. Since all the transmissions in the WMN are routed to and from the gateway, high concentration of traffic at the gateway leads to saturation. This may cause packet drops at GW, which is highly inefficient especially after being consumed a lot of network resources.

The power control [12] is a fundamental issue of $\mathrm{WMN}$ as it reduces nodes power consumption as well as it increases the number of successful transmission by decreasing multi-user interference. The channel spatial reuse can be improved by scheduling more wireless nodes to be transmitted simultaneously, thus increasing the throughput. However, to guarantee the required Signal-to-Interference plus Noise Ratio (SINR) for each receiver the wireless nodes need to increase the transmission power. The transmission power can be reduced at the cost of poor network throughput. This can be achieved by making the neighboring nodes the currently active node to be silent. Scheduling that concerns about only 
the throughput maximization of network may force some links to receive very low bandwidth allocation or cause some links to remain silent all the time to prevent interference. High bandwidth utilization leads to high network throughput.

Multiple access interference is a major issue in wireless communication systems. Interference leads to both unfair channel allocations on IEEE 202.4 MAC protocol and packet drops when the SINR falls below the packet reception threshold of receiver node. A cycle of a schedule is the time needed to activate all the links in WMN according to the scheduling algorithm used. As the cycle length decreases the network throughput increases.

\section{SCHEDULING}

\subsection{Overview}

Scheduling is one of the important factors that have a great impact on the system performance. Scheduling is to schedule resources (channels, mini-slots) to serve different nodes in the network. It is a sequence of fixed length time slots. Each possible transmission is assigned a time-slot in such a way that even if several transmissions are assigned to the same time slot, it must not collide. There are two kinds of scheduling: node scheduling and link scheduling.

In node scheduling $[1,12,10,16]$, also known as broadcast scheduling, the entities scheduled are the nodes itself. The transmission of a node must be received by all of the neighbouring nodes collision free.

In a link scheduling $[2,4,3,6,7,9,12]$, the links between the nodes are scheduled. The transmission of a node must be received by a particular neighbour node without collision at the receiver.

A good scheduling ensures high end-to-end throughput on routers and hence end-to-end data rate. A fair scheduling could gradually increase network capacity, bandwidth utilization and ensures fairness among different nodes.

\subsection{Scheduling Mechanisms}

Scheduling schemes can be defined as both centralized and distributed. A scheduling algorithm has to allocate the resources to the users according to their needs.

A centralized approach could be taken if the gateway is controlling the scheduling $[1,2,3,5,7,9,10,13]$ and the scheduled packets are transmitted in a collision free manner, eg, STDMA.

A distributed approach relies on the assumption that all the mesh routers in the network will co-operate $[4,6,12,16]$. Each MR itself decides whether to send or hold off the packets and to give opportunity to send to other MR. The lack of central authority avoids the single point of failure problem.

\subsection{Modeling Schemes}

Scheduling is to schedule resources (channels, mini-slots) to serve different nodes in the network. It is a sequence of fixed length time slots. Each possible transmission is assigned a time-slot in such a way that even if several transmissions are assigned to the same time slot, it must not collide. The modelling schemes include interference-based and tree-based modelling. Description is given below.

\subsubsection{Interference-Based Model}

Because of the shared medium access nature of channels, the multi-user interference is widely recognized in system design perspective. Multi-user interference is unavoidable and its impact on system performance is diverse. Interference could degrade the throughput of wireless mesh networks. This performance degradation is caused due to two main reasons: firstly, sources may send more traffic than what the network can support. Additional traffic reduces the capacity of bottleneck links. Secondly, the current protocols are unable to accurately measure link and path quality for the path selection purpose [11]. Two nodes can form a communication link only if the Signal-to-noise Ratio (SNR) is not less than communication threshold.

Interference models find nodes or links that can transmit simultaneously by assigning same time-slot, provided they do not interfere with each other. The wireless interferences can be classified into two main categories: primary and secondary interference.

Primary interference occurs when a node has to do more than one work in single time slot. Primary interference accounts to self-interference (a node cannot transmit and receive at the same time) [11,13], collision (two nodes cannot transmit at the same time)[6], multicast (a node can transmit data only to one node in a time slot)[1]

Secondary interference occurs when different pair of nodes transmits simultaneously $[1,2,7]$. Two main interference models include protocol and physical interference model.

Protocol interference model shows the characteristics similar to CSMA/CA $[11,14]$. It is not much preferred because it achieves fairness at the cost of network throughput. This model does not allow power control. And also it does not take into account the aggregate interference caused by all transmissions.

In physical interference model $[11,12,14]$, the transmission between two nodes is possible only when the SINR at the receiver node is greater than a threshold value. Its characteristic is suited with STDMA access scheme.

Some of the interference-based scheduling schemes are discussed below.

\subsubsection{Interference Based Scheduling Algorithms}

IAFS [1], a TDMA like MAC solution, improves the fairness of multicast service in WMNs. It takes into account the interference and fading among multicast nodes and assigns transmission time slots while maximizing the spatial reuse achieving high throughput. This scheduling scheme consists of four components: the tree construction, the interference model, the scheduling algorithm, and schedule propagation. SSP (Success Probability Product) is used as the tree construction metric. A threshold-based model is used which helps in finding nodes that can transmit simultaneously. This leads to reduced cycle length, gradually increased sending rate and throughput. However, it also leads to unfairness. The scheduling algorithm consists of three phases: compatibility matrix $(\mathrm{CM})$ and graph construction, clique enumeration and clique selection. CM describes if pair of nodes can transmit simultaneously. After the CM construction phase, all possible cliques in the graph are enumerated. A clique is set of links that can all be enabled at the same time. LOP (Least Overlapped First) metric is used so that each clique is assigned a rank. Then that clique is selected which has the smallest rank, among the cliques of the same size. After computing the schedule, the root node propagates it to other nodes.

A collision-free centralized scheduling algorithm, TTS algorithm [7] has been proposed by Bo Han to provide high quality wireless multimedia services. This algorithm considers 
important performance metrics such as fairness, channel utilization and transmission delay. In this algorithm each mesh node is assigned a service token, which is used to allocate time slots to each link based on the traffic demand of the link's transmitter. This mechanism avoids node starvation thereby the fairness is guaranteed. A link is marked as available, if the service token of the transmitter of a link is non-zero otherwise, it is marked as idle. An available link can be marked as scheduled (in the current time slot) and interfered (conflicting neighboring links) based on some criterion. The criteria in this work includes: random (the scheduled link is selected randomly each time), min interference (only those links are chosen for scheduling whose transmitter interferes the minimal number of other nodes), nearest to BS and finally farthest to BS (those links are selected that has the minimal or maximal hop count to the BS). Node ID is used to break the tie in criteria.

ALS algorithm [10] has been proposed by Peng Du, Wenyan $\mathrm{Lu}$. In this scheme each node has one transceiver that can be tuned between multiple channels. It eliminates the secondary interference thereby reducing the length of scheduling. The primary interference cannot be avoided as only one transceiver is supported on each node. One node cannot support multiple channels simultaneously. However, in order to avoid secondary interference different nodes may operate on different channels. In ALS each node is assigned a token. A link is considered as 'available' whose transmitter has a non-zero token. A NS algorithm is used to find a group of links to transmit simultaneously from the available links and channel is assigned to them.

Nguyen H. Tran, Choong Seon Hong [8] proposed a greedy algorithm that schedule links fairly in WMNs under physical interference model by using STDMA scheme. In this work the whole network is not considered, instead a sub-graph is investigated. By this the border links are not starved any more as the higher priority links (near to gateway) has been reduced. Minimum Spanning Tree (MST) is chosen as the sub-graph of the network graph. Throughput and fairness are addressed in this work.

\subsubsection{Tree-Based Modeling}

The whole network is reduced into a tree model. The schedule can be obtained by studying the set of edges. The network can be represented as a tree with a set of nodes $\mathrm{V}$ and a set of edges E. Interferences from other nodes are not taken into account. Two edges can be assigned the same time slot if and only if the nodes connected to the edges are all mutually distinct as a node cannot receive and transmit simultaneously in the same slot. And also a node cannot receive a packet while neighboring nodes are transmitting. However the above criterions are not sufficient to guarantee that the assignment is collision-free. They may not be able to transmit simultaneously. Some of the tree based scheduling algorithms are discussed below.

\subsubsection{Tree-Based Scheduling Algorithms}

A joint scheduling and routing algorithm [9] maximizes the utilization of the network. It also guarantees the fairness. In this routing algorithm the network capacity is maximized by selecting highest end-to-end data rate paths. It generates best schedule on the selected links for maximizing network utilization. The interference is not addressed in this work. It aims at maximizing throughput capacity.

The joint scheduling and power control scheme [12] addresses the power control issue for supporting multicast traffic. This algorithm provides an optimal solution to power control problem for a given set of senders and receivers. This distributed algorithm provides sub-optimal solution to power control problem since it is based on local information. It maximizes the number of successful multicast transmissions. This algorithm calculates the sender's power level. It aims at meeting the SINR requirements at any receiving nodes while minimizing the total power consumption.

A novel interference-aware route construction algorithm and an enhanced centralized mesh scheduling scheme [2] achieves high throughput utilizing the WiMax mesh networks. The traffic load demand and interference conditions are considered. This scheme creates a tree-based routing framework that along with scheduling is interference aware and leads to better spatial spatial-reuse and thus higher spectral efficiency. The concept of blocking metric $\mathrm{B}(K)$ is used to model the interference level of routes in the mesh.

A fair scheduling mechanism [6] optimizes the bandwidth utilization in the mesh network. This scheme also ensures perclient fairness. It is a collision free solution that assigns transmission rights to the links in the $\mathrm{WMN}$ and maximizes the spatial reuse. The scheme includes construction of compatibility matrix, graph, construction clique and schedule update.

\section{LOAD BALANCING}

In WMN, a subset of nodes called IGW acts as the central point of connectivity to wired infrastructure. The traffic in WMN is routed to and from the IGW. Because of the tremendous increase in the traffic in WMN and also due to the limited link capacity, the gateway is likely to be a potential bottleneck. Thus load balancing has become an important issue in WMN. Saturation at gateways due to high traffic can lead to packet loss, which in turn affects the system performance. Thus it is necessary to balance the traffic load over all GWs in order to alleviate the congestion. This is possible by switching the point of attachment of an active source serviced congested gateway to under-utilized gateways. An efficient load balancing mechanism helps in avoiding network congestion and also increases the efficiency of network resource utilization. The classification of load balancing schemes is shown in fig 1 .

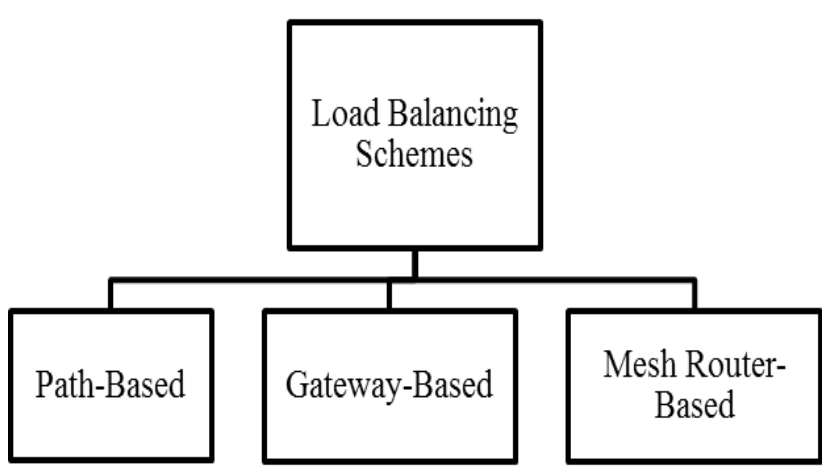

Fig 1: Load Balancing Schemes

\subsection{Load Balancing Techniques}

TeXCP [15] is a distributed traffic engineering protocol that balances load in real-time. It uses multiple paths to deliver ingress to an egress router demands. Each TeXCP agent incorporated in the ingress router runs a load balancer that slits the traffic of an ingress-egress pair among its available 
paths. It optimizes the maximum utilization and balances the load.

LARM [16], routing metric is proposed for multi radio mesh networks. This metric is incorporated into load balancing routing protocol, called LBM. LBM selects the route with less congestion, low packet loss, high data rate and low level of interference. The node on the active route finds the alternative least route to the destination on detecting a break on the active route. This scheme results in high packet delivery percentage, throughput and lower delay on high traffic load.

LDBAL [17], a Queue based Load Balancing scheme have been proposed as a part of congestion aware load-balancing scheme that includes two phases: 1) Gateway discovery protocol, where all the nodes discover their primary gateways. This is achieved using a table of gateway IDs at the nodes. 2) Load migration procedure, where the load is migrated to nearby less congested gateways by maintain a queue at each gateways. This scheme results in improved PDR and throughput. As soon as the GW detects congestion it informs the active mesh routers to switch to other GW. Thus the congestion at any GW is reduced.

GWLB [18], a distributed load balancing protocol where the GWs coordinate to reroute flows from congested to underutilized GWs. This scheme addresses the effects of interference. Re-routing the traffic from overloaded domain to less congested domain leads to better capacity utilization and throughput. The overload of both domains is reduced, where a domain is a set of nodes served by a GW.

CWB [19], considers both traffic load and interference to provide load balancing in WMNs. It provides load balancing features at mesh routers and supports global load-aware routing. It helps the routing protocol to balance the traffic and improve the network capacity, thereby avoiding routing traffic through congested areas. This scheme results in high network throughput and low packet delay even during high traffic loads.

WCETT-LB [20], introduces load balancing feature at the mesh routers. It provides a congestion aware routing and traffic splitting mechanism in order to achieve global load balancing in the network. Using an average queue length at each node and comparing it with a threshold value the congestion level can be evaluated. This scheme provides a mechanism to handle the interference between the nodes. Since each node is determining its own congestion level the communication overhead is reduced. Even at high network load the proposed scheme results in high network throughput and lower end-to-end delay.

\section{ANALYSIS}

Several scheduling mechanisms like link or node scheduling centralized or distributed, use of single or multiple channels for traffic and data, medium access schemes have been discussed in Table. 1.

Table. 2 show a comparison of interference and tree based schemes. IAFS [1] does not achieve 100 percent throughput, but still can provide high fairness and medium packet delivery ratio. A small cycle length leads to high throughput. TTS [7] achieves high fairness at the cost of throughput. ALS [10] provides less delay, but medium throughput and fairness. Greedy algorithm [8] provides high throughput and fairness. Joint scheduling and routing algorithm [9] gives medium packet delivery ratio with high throughput and fairness and less power consumption. Joint scheduling and power control [5] achieves medium throughput and less power consumption with lower packet delivery ratio. Interference aware route construction [2] caused medium packet delivery ratio but high throughput. The channel utilization is high. Fair scheduling mechanism [6] causes only a medium packet delivery ratio, but achieves high throughput and fairness. The interference model seems more beneficial compared to tree-based as it provides an average of better throughput, fairness, channel utilization and lower power consumption.

Three load balancing schemes have been analyzed in Table. 3 . Among the path-based scheme LARM [16] performs much better than TeXCP [15]. The parameters throughput, packet delivery ratio are high in LARM. In gateway-based, GWLB [18] is better than LDBAL [17]. Even though the delay is low in LDBAL, the throughput and packet delivery ratio are low for LDBAL. The overhead is high too. In mesh router-based scheme CWB [19] outperforms WCETT-LB [20] in terms of throughput, delay and channel utilization. The gateway-based scheme achieve optimum load balance with low overhead, less interference effects, high throughput, better channel utilization and high packet delivery ratio.

\section{Table 1. Comparison of Scheduling Mechanisms}

\section{Schemes}

$\begin{array}{llllll}\text { References } & \text { Link/ } & \text { Centralized/ } & \text { Single/ } & \text { Medium } & \text { Interference } \\ \text { Node } & \text { Scheduling } & \text { Decentralized } & \text { Multi Channel } & \text { Access } & \text {. }\end{array}$

\section{Koutsonikolas, node [1] \\ (1)}

W. Jia [7]

Peng DU [10]

N. H. Tran [8]

link

link

link
Decentralized

centralized

Collision free

centralized

multi

multi

single

centralized
Spatial TDMA

TDMA

TDMA
Spatial TDMA
Threshold-based

Eliminates primary interference Eliminates secondary interference

Physical model 


\begin{tabular}{|c|c|c|c|c|c|}
\hline S. Nahle [9] & link & centralized & single & TDMA & Reduce interference \\
\hline K. Wang [5] & node & distributed & single & TDMA & $\begin{array}{l}\text { Decrease multi-user } \\
\text { interference }\end{array}$ \\
\hline H. Y. Wei [2] & link & centralized & single & TDMA & $\mathrm{n} / \mathrm{a}$ \\
\hline N. B. Salem [6] & link & distributed & multi & Spatial TDMA & $\mathrm{n} / \mathrm{a}$ \\
\hline
\end{tabular}

Table 2. Comparison between different Scheduling Algorithms

\begin{tabular}{|c|c|c|c|c|c|c|c|c|}
\hline \multirow[b]{2}{*}{ Types } & \multirow[b]{2}{*}{$\begin{array}{l}\text { Modelling } \\
\text { Schemes }\end{array}$} & \multicolumn{7}{|c|}{ Parameters } \\
\hline & & $\begin{array}{c}\text { Packet } \\
\text { Delivery } \\
\text { Ratio } \\
\end{array}$ & Delay & Throughput & Fairness & $\begin{array}{c}\text { Cycle } \\
\text { Length }\end{array}$ & $\begin{array}{c}\text { Power } \\
\text { Consumptio } \\
\mathbf{n}\end{array}$ & $\begin{array}{l}\text { Channel } \\
\text { Utilization }\end{array}$ \\
\hline \multirow{4}{*}{$\begin{array}{l}\text { Inter } \\
\text { ference- } \\
\text { Based }\end{array}$} & IAFS[1] & Medium & $\mathrm{n} / \mathrm{a}$ & Not $100 \%$ & High & Small & $\mathrm{n} / \mathrm{a}$ & $\mathrm{n} / \mathrm{a}$ \\
\hline & TTS [7] & High & $\begin{array}{l}\text { Nodes at higher } \\
\text { level causes } \\
\text { more delay }\end{array}$ & Low & High & $\begin{array}{l}\text { Reduced } \\
\text { length }\end{array}$ & Not an issue & $\begin{array}{l}\text { Is increased } \\
\text { with the } \\
\text { number of } \\
\text { nodes. }\end{array}$ \\
\hline & ALS [10] & Medium & $\begin{array}{l}\text { Low; but } \\
\text { increases with } \\
\text { more nodes. }\end{array}$ & Medium & Very less & $\begin{array}{c}\text { Small; } \\
\text { but } \\
\text { increases } \\
\text { with } \\
\text { number } \\
\text { of nodes }\end{array}$ & $\mathrm{n} / \mathrm{a}$ & $\mathrm{n} / \mathrm{a}$ \\
\hline & GA [8] & $\mathrm{n} / \mathrm{a}$ & $\mathrm{n} / \mathrm{a}$ & $\begin{array}{c}\text { Increases with } \\
\text { multi radios }\end{array}$ & $\begin{array}{c}\text { Fair for all } \\
\text { nodes }\end{array}$ & $\mathrm{n} / \mathrm{a}$ & $\mathrm{n} / \mathrm{a}$ & $\mathrm{n} / \mathrm{a}$ \\
\hline \multirow{4}{*}{$\begin{array}{l}\text { Tree- } \\
\text { Based }\end{array}$} & JSR[9] & Medium & $\mathrm{n} / \mathrm{a}$ & High & Fair & $\mathrm{n} / \mathrm{a}$ & Low & High \\
\hline & JSP[5] & Low & $\mathrm{n} / \mathrm{a}$ & Medium & $\mathrm{n} / \mathrm{a}$ & $\mathrm{n} / \mathrm{a}$ & Low & $\mathrm{n} / \mathrm{a}$ \\
\hline & IARC[2] & Medium & Low & $\begin{array}{l}\text { High; but } \\
\text { decrease with } \\
\text { number of } \\
\text { nodes }\end{array}$ & Very less & $\mathrm{n} / \mathrm{a}$ & $\mathrm{n} / \mathrm{a}$ & High \\
\hline & FSM [6] & Medium & Low & High & High & Small & $\mathrm{n} / \mathrm{a}$ & High \\
\hline
\end{tabular}


Table 3. Comparison of Load Balancing Schemes

\section{Parameters}

\begin{tabular}{|c|c|c|c|c|c|c|c|}
\hline $\begin{array}{l}\text { Load } \\
\text { Balancing } \\
\text { Schemes }\end{array}$ & Types & Overhead & Interference & Throughput & Delay & $\begin{array}{l}\text { Channel } \\
\text { Utilization }\end{array}$ & $\begin{array}{l}\text { Packet Delivery } \\
\text { Ratio }\end{array}$ \\
\hline \multirow{2}{*}{$\begin{array}{l}\text { Path- } \\
\text { Based }\end{array}$} & TeXCP & $\mathrm{n} / \mathrm{a}$ & $\mathrm{n} / \mathrm{a}$ & $\mathrm{n} / \mathrm{a}$ & Low & $\begin{array}{c}\text { Lower } \\
\text { compared to } \\
\text { LARM }\end{array}$ & $\mathrm{n} / \mathrm{a}$ \\
\hline & LARM & $\mathrm{n} / \mathrm{a}$ & $\begin{array}{c}\text { Reduced } \\
\text { interference(intra/inter) } \\
\end{array}$ & High & $\begin{array}{l}\text { Very } \\
\text { low }\end{array}$ & Medium & High \\
\hline \multirow{2}{*}{$\begin{array}{l}\text { Gateway- } \\
\text { Based }\end{array}$} & LDBAL & $\begin{array}{l}\text { High } \\
\text { compared }\end{array}$ & $\mathrm{n} / \mathrm{a}$ & $\begin{array}{l}\text { Low compared } \\
\text { to GWLB }\end{array}$ & Low & $\mathrm{n} / \mathrm{a}$ & $\begin{array}{c}\text { Low compared to } \\
\text { GWLB }\end{array}$ \\
\hline & GWLB & low & $\begin{array}{l}\text { Avoids negative effects of } \\
\text { interference(intra) }\end{array}$ & High & $\mathrm{n} / \mathrm{a}$ & $\begin{array}{c}\text { High } \\
\text { compared to } \\
\text { LDBAL }\end{array}$ & High \\
\hline \multirow[t]{2}{*}{$\begin{array}{l}\text { Mesh } \\
\text { Routers- } \\
\text { Based }\end{array}$} & CWB & $\begin{array}{c}\text { High } \\
\text { compared } \\
\text { to } \\
\text { WCETT- } \\
\text { LB }\end{array}$ & $\begin{array}{l}\text { Performance not affected by } \\
\text { interference. }\end{array}$ & $\begin{array}{l}\text { High compared } \\
\text { to WCETT-LB }\end{array}$ & $\begin{array}{l}\text { Very } \\
\text { low }\end{array}$ & High & $\mathrm{n} / \mathrm{a}$ \\
\hline & $\begin{array}{l}\text { WCETT- } \\
\text { LB }\end{array}$ & low & $\begin{array}{l}\text { Reduce the effect of } \\
\text { interference(intra/inter) }\end{array}$ & Medium & Low & $\mathrm{n} / \mathrm{a}$ & $\mathrm{n} / \mathrm{a}$ \\
\hline
\end{tabular}

\section{CONCLUSION}

In this paper, the problem of scheduling and load balancing in Wireless Mesh Networks has been discussed. The classification of scheduling models are presented and elaborated which includes interference-based and tree-based. The path-based, gateway-based and mesh router-based load balancing schemes have been explored. The efficiency and performance of each scheme have been discussed. On the basis of the comparison the interferencebased and gateway-based schemes are found to be more beneficial as these schemes provide better throughput, fairness, channel utilization, high packet delivery ratio, less delay and less power consumption compared to others.

\section{REFERENCES}

[1] D. Koutsonikolas, S.M. Das, Y.C. Hu,( March 2008) “An interference-aware fair scheduling for multicast in wireless mesh networks", Journal of Parallel and Distributed Computing 68, pp. 372-386.

[2] H.-Y. Wei, S. Ganguly, R. Izmailov, Z.J. Haas, (June 2005 ) "Interference-aware IEEE 802.16 WiMax mesh networks", in: Proc. of IEEE 61st Vehicular Technology Conf. (VTC 2005), vol. 5, pp. 3102-3106.

[3] J. B. Ernst , M. K. Denko, (2011) "The design and evaluation of fair scheduling in wireless mesh networks", Journal of Computer and System Sciences 77, pp. 652-664.

[4] J. Tang, G. Xue, C. Chandler, W. Zhang, (May 2006) "Link scheduling with power control for throughput enhancement in multi-hop wireless networks", in: IEEE Transactions on Vehicular Technology, vol. 55, pp. 733-742.

[5] K. Wang, C.F. Chiasserini, R.R. Rao, J.G. Proakis, (2003)“A distributed joint scheduling and power control algorithm for multicasting in wireless ad hoc networks", in: Proc. of IEEE Int. Conf. on Communications, pp. 725-731.

[6] N.B. Salem, J.-P. Hubaux, (2005 ) "A fair scheduling for wireless mesh networks", in: Proc. of 1st IEEE Workshop on Wireless Mesh Networks (WiMesh 2005).

[7] B. Han, W. Jia, and L. Lin, (2007) "Performance evaluation of scheduling in IEEE 802.16 based wireless mesh networks", Computer Communications, pp. 782-792.

[8] N. H. Tran, C. S. Hong, "Fair Scheduling for Throughput Improvement in Wireless Mesh Networks", pp. 1310-1312.

[9]S.Nahle,N.Malouch,(2008)"Joint routing and scheduling for maximizing fairthroughput in WiMAX mesh network", IEEE 19th International Symposium on PIMRC, pp. 1- 5.

[10]Peng Du, Weijia Jia, Liusheng Huang, Wenyan Lu(2007) "Centralized Scheduling and Channel Assignment inMultiChannel Single Transceiver WiMax MeshNetwork", in IEEE Wireless Communications and Networking Conference, pp. $1734-1739$.

[11] Y. Li et. al. Effects of interference on wireless mesh networks: Pathologies and a preliminary solution", in Proceedings of HotNets. 
[12] D. Lertpratchya,D. M. Blough, "Exploring the Design Space of Greedy Link Scheduling Algorithms for Wireless Multihop Networks", School of Electrical and Computer Engineering Georgia Institute of Technology, 2011.

[13] P. Wang, S. Bohacek, "Tractable Computation of Optimal Schedules and Routing in Wireless Mesh Networks", Citeseer.

[14] P. Gupta, P. R. Kumar, "The Capacity of Wireless Networks", IEEE Transactions on Information Theory, vol. 46, NO. 2, March 2000.

[15] S. Kandula, D. Katabi, B, "Walking the tightrope: Responsive yet stable traffic engineering", in Proc. ACM SIGCOMM, Aug. 2005.

[16] A. Le, D. Kum, Y. Cho, and C. Toh, "Routing with LoadBalancing in Multi-Radio Wireless Mesh Networks", IEICE TRANS. COMMUN., No. 3, 2009.
[17] D. Nandiraju, L. Santhanam, N. Nandiraju, D.P. Agrawal, "Achieving load balancing in wireless mesh networks with multiple gateways", in: Proceedings of IEEE International Workshop on Wireless Mesh-Networks and Applications (WiMa06), 2006

[18] Galvez,J.J, Ruiz,P.M.; Skarmeta, "A distributed algorithm for gateway load-balancing in Wireless Mesh Networks", Wireless Days, 2008, pp. 1- 5.

[19] L. T. Nguyen, Beuran R, ShinodaY, "A loadaware routing metric for wireless mesh networks", in: proc. of IEEE symposium on Computers and Communications, 2008 , pp. $429-435$.

[20] Liang Ma, Denko M.K, "A Routing Metric for Load Balancing in Wireless Mesh Networks", in: proc. of IEEE

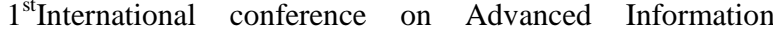
Networking and Applications Workshops, 2007, pp. 409 414. 